HID 47 (2020)

\title{
EL EMPRÉSTITO DE SEVILLA A JUAN II EN $1429^{1}$
}

THE FORCED LOAN FROM SEVILLE TO JOHN II IN 1429

\section{Antonio Collantes de Terán SÁnchez}

Real Academia Sevillana de Buenas Letras

acdets@hotmail.com

RESUMEN: Uno de los instrumentos utilizados por los monarcas castellanos para hacer frente a necesidades extraordinarias fue el crédito. Una de las formas en que se manifestó fue el empréstito o préstamo forzoso demandado a los súbditos, sobre el que los trabajos son todavía escasos. En este se analiza el demandado por Juan II en 1429 al concejo de Sevilla, cuya recaudación fue muy conflictiva por los rechazos a los distintos repartimientos que efectuaron tanto la hacienda real como las autoridades municipales.

PALABRAS CLAVES: Empréstito; crédito; hacienda; Sevilla; Castilla; Juan II; 1429.

ABSTRACT: Credit was one of the instruments used in the late Middle Ages by the Castilian monarchs to cope with extraordinary needs. One of the ways in which it manifested itself was the "empréstito" (forced loan) demanded of the subjects, on which the related papers are still scarce. This paper discusses the credit that John II required to the council of Seville in 1429, whose collection was very controversial due to the rejections of the different distributions made by both the royal treasury and the municipal authorities.

KEYWORDS: Empréstito (forced loan); credit; public finances; Seville; Castille; John II; 1429.

Recibido: 9-3-2020; Aceptado: 2-7-2020; Versión definitiva: 2-7-2020

1. Abreviaturas utilizadas: AMS $=$ Archivo Municipal de Sevilla; $\mathrm{AGS}=$ Archivo General de Simancas; $\mathrm{d} .=$ dinero; $\mathrm{f} .=$ folio; leg. $=$ legajo; $\mathrm{mrs} .=$ maravedís $; \mathrm{n}^{\mathrm{o}}=$ número; $\mathrm{p} .=$ página; $\mathrm{PM}=$ Papeles del Mayordomazgo; Sec. $=$ Sección.

Este trabajo forma parte del Proyecto de Investigación del Programa Estatal de Generación del Conocimiento y Fortalecimiento Científico y Tecnológico del Sistema de I+D "La construcción de una cultura fiscal en Castilla: poderes, negociación y articulación social (ca. 1250-1550)" (PGC2018097738-B-100), integrado en la Red Arca Comunis.

Copyright: (C) Editorial Universidad de Sevilla. Este es un artículo de acceso abierto distribuido bajo los términos de la licencia de uso y distribución Creative Commons Reconocimiento-NoComercialSinObraDerivada 4.0 (CC BY-NC-ND 4.0) 
Los cuantiosos recursos que necesitó Juan II para emprender la guerra contra los reyes de Aragón y de Navarra le obligó a adoptar una serie de medidas extraordinarias, entre ellas recurrir al préstamo de plata y de dinero. Lo que, posiblemente, en un primer momento fue un recurso que afectó a las instituciones eclesiásticas y a ciertos miembros de las élites socioeconómicas ${ }^{2}$, acabaría implicando a otros sectores sociales a través de su demanda a los concejos, con el fin de ampliar o sustituir el proyecto inicial. Al menos, esto es lo que ocurrió en Sevilla ${ }^{3}$.

A juzgar por el desarrollo del proceso de recaudación, parece que el punto de partida fue la petición de 2.000.000 de maravedís que el monarca hizo a 43 vecinos en concepto de préstamo. Estos figuran en un documento sin fecha, encabezado como "El repartimiento"4. La relación, que fue confeccionada en la corte ${ }^{5}$, es posible que la trajera el tesorero real Nuño López de Saldaña, veinticuatro de Sevilla y alcaide de sus Atarazanas, pues en la carta de poder, fechada el 24 de junio, manifiesta el monarca que lo enviaba como recaudador de las doblas, florines, maravedís, oro y plata que cualquier persona prestase, ya fuese prelado, clérigo, caballeros o cabildo ${ }^{6}$. La relación responde a lo indicado ${ }^{7}$. Las cantidades están expresadas en doblas y la encabeza el arzobispo, con 3.000, al que siguen dos canónigos con 1.000 cada uno; cantidad asignada también a otras siete personas de distinta condición social. Además, están incluidos tres veinticuatros o regidores (con 800 cada uno) y cuatro jurados (con 600 y 1.000). Con todo, el colectivo más numeroso es el de los traperos/mercaderes, con nueve y dos miembros respectivamente, y unas cantidades que oscilan entre las 300 y las 800 doblas. Hay varios con oficios vinculados a actividades financieras y hacendísticas, entre otros, dos cambiadores y un corredor. Además, hay nueve sin indicación de actividad, pero que probablemente habría que incluir entre los citados - de los que alguno era

2. Se constata en las cartas de poder de sus enviados a Murcia y Sevilla para tratar de la operación, la de Pedro Álvarez de Arévalo (Abellán Pérez 1984, pp. 397-398) y la del tesorero Nuño López de Saldaña (AMS, Sec. 15, PM, 1429-1430, n 138, ff. 3r-4v), pero también en las relaciones de ingresos y gastos de zonas del reino, en las que figuran entregas de particulares (AGS, EMR, leg. 1, f. 99r-102v), y en las actas del concejo de Burgos, donde se alude a los 2.000 marcos de plata solicitados a los mercaderes, más los 200.000 mrs. a repartir entre los vecinos (Guerrero Navarrete 1999, pp. 99,100; MacKay, 2006, pp. 129, 130). Sobre la demanda a instituciones eclesiásticas, Ortego, 2012, pp. 151,152 .

3. A esta operación aludí en su día en un trabajo de conjunto sobre el empréstito en Sevilla (2009), pero el volumen de documentos que se conserva -muy superior al de los restantes-, y su complejidad, aconsejaba dedicarle un trabajo específico, para permitir profundizar mejor en su gestión, así como en los entresijos de las relaciones entre los concejos y las necesidades regias de recurrir al crédito. Estas cuestiones, entre otras, se han planteado en trabajos posteriores, con especial atención a los empréstitos de finales del siglo XV, como los de Ortego Rico 2015; Gálvez Gambero y Triano Milán 2016; Triano Milán 2018.

4. AMS, Sec. 15, PM, 1429-1430, nº 138, f. 20r-v. Apéndice 1.

5. En una reclamación por las cantidades que se les demandaban, el primer argumento que esgrimen varias personas que figuraban en una relación especial es que "ellos nin algunos dellos non eran de aquellos a quien el rey nuestro sennor auía escripto en especial que le prestasen maravedís nin doblas algunas" (AMS, Sec. 15, PM, 1429-1430, nº 138, f. 256r).

6. AMS, Sec. 15, PM, 1429-1430, nº 138, f. 3r.

7. Apéndice 1 . No he podido averiguar a que corresponden las cantidades que figuran en la columna de la izquierda. 
familiar-, como el mayordomo del concejo de 1423, y apellidos con protagonismo en los arriendos de impuestos, varios de ellos conversos. En fin, cinco vecinos de villas del alfoz: el vicario de Sanlúcar la Mayor, un jurado de Alcalá de Guadaira y tres de Utrera, de los que dos son personas cualificadas, un escribano y Juan Alonso de Montesdoca, apellido que remite a un linaje de la élite local. A este heterogéneo grupo se le asignaron cantidades que oscilaron entre las $300 \mathrm{y}$ las 1.000 doblas.

La petición generó un importante rechazo, y no se llevó a efecto. Ante lo cual, el concejo decidió repartir los dos millones entre el conjunto de los vecinos y moradores de la ciudad y del alfoz, pero configurando con algunos de ellos una nómina específica, que los documentos denominan "personas especiales"8. Esta decisión resulta extraña, porque a la mayoría se le asignaron cantidades similares a las que se encuentran en las relaciones de los vecinos que entraron en el reparto general por collaciones y pueblos. A su vez, entre estos aparecen unos pocos con cantidades altas que se podían considerar como personas especiales ${ }^{9}$.

La comparación entre las dos relaciones - la de las doblas y la de personas especiales- es muy instructiva de los cambios que se introdujeron. En primer lugar, han desaparecido los eclesiásticos y oficios públicos (veinticuatros y jurados); en segundo lugar, se han reducido considerablemente las cantidades solicitadas. Entre las personas especiales aparecen diecisiete que ya figuraban en la de las doblas, por lo que se pueden comparar las peticiones de uno y otro momento ${ }^{10}$. Estas diecisiete sumaban 10.000 doblas, que traducidas a maravedís alcanzaban la cifra de 710.000 mrs. $^{11}$; ahora, con el nuevo reparto estos mismos deberían prestar 150.000 mrs.; es decir, la quinta parte de aquella cantidad.

Cuarenta y siete son las personas especiales ${ }^{12}$, con un destacado protagonismo de los comerciantes y artesanos, en su mayoría ligados al textil (traperos, mercaderes, cambiadores, sastres, roperos, sayaleros, lenceros $)^{13}$, aparte de un corredor, un monedero, etc. La media de las cantidades que debían aportar fue de $6.117 \mathrm{mrs}$. Las más altas correspondieron al jurado de Alcalá de Guadaíra (30.000 mrs.), a un pertiguero (¿de la Catedral?) (25.000 mrs.), a tres personas que no indican oficio

8. Apéndice 2.

9. Entre ellos, Diego López Bonifante, $v^{\circ}$ S. Nicolás, y Diego Sánchez de Robleda, trapero, $v^{\circ}$ Barrio de Francos, con 15.000 mrs. cada uno; Pedro Fernández de Ríos (¿), v ${ }^{\circ}$ S. Pedro, y Alfonso Martínez de Martín Cabrera, $v^{\circ}$ S. Martín, con 10.00 mrs. cada uno; la viuda del trapero Pedro Ximénez, el viejo, quien había tenido un papel importante en la gestión de los asuntos económicos del concejo, $\mathrm{v}^{\mathrm{a}}$ Salvador, con 8.000 mrs. (AMS, Sec. 15, PM, 1429-1430, nº 138, ff. 48, 58, 169).

10. Apéndice 1.

11. Los documentos no especifican qué tipo de dobla. De las tres que corrían por estas fechas en Sevilla (morisca o corriente, baladí y castellana) he utilizado el valor de la primera, que parece la más empleada y la que por su equivalencia (71 mrs. la dobla) se aproxima más al número de doblas repartido (Collantes de Terán Sánchez 2000, pp. 63, 75).

12. En ocasiones, en la cantidad asignada se incluyen varios miembros de la familia (hermanos, madres, hijos), por lo que el número real es algo mayor, pero no los he tenido en cuenta pues, salvo un caso, no es posible conocer si tenían independencia económica.

13. AMS, Sec. 15, PM, 1429-1430, no 138, ff. 6r-7v. Algo similar a lo que ocurrió en Burgos y Toledo (Guerrero Navarrete 1999, p. 100. AGS, EMR, leg. 1, ff. 99r-100v). 
(15.000 y 10.000 mrs.), el resto está por debajo de estos valores, siendo la cantidad mínima 1.500 mrs. El total de lo que debían aportar ascendió a 287.500 mrs.; es decir, el 14,4\% de los dos millones, por lo que el 85,6 \% restante se repartió entre los vecindarios de la ciudad y de los pueblos.

No hay muchos datos sobre los criterios empleados para llevar a cabo el citado reparto. Aunque algunos están claros. La unidad fiscal en la ciudad fue la collación o el barrio, en total 32; en el alfoz las localidades que lo integraban, aunque no todas, porque en los cargos de los recaudadores figuran 39 de las $53^{14}$, que lo componían. Las que faltan, la mayoría de la Sierra de Aroche, son, en general, entidades pequeñas, que precisamente por ello quizá quedaron excluidas, aunque alguna posiblemente también por gozar de exenciones para favorecer su poblamiento. A cada una de estas unidades se le asignó una cantidad a repartir entre sus vecinos y moradores, siguiendo con toda probabilidad los criterios empleados para la confección de los padrones de cuantía para el pago de los pedidos, si no es que se utilizaron directamente dichos padrones, y estableciéndose unas cantidades mínimas y máximas a prestar. Por tanto, se tuvo en cuenta el número de vecinos y sus patrimonios. Así, en el primer reparto se observa que el número de prestamistas de cuatro de las collaciones más pobladas está por debajo de la media de los prestamistas por collación, que fue de dieciséis.

Aparte de esa posibilidad, también se puede conocer algo de quiénes quedaron exentos. Por un lado, los considerados pobres; por otro, los eclesiásticos, los miembros de la nobleza y aristocracia, personas que vivían con ellos -aunque aparecen algunas de sus mujeres y/o viudas e hijos-, en fin, algunos oficios y cargos públicos, como los jurados y los veinte alguaciles a caballo, según se deduce de distintas cartas remitidas por los diputados a los recaudadores. Sí figuran en las relaciones algunos extranjeros, entre ellos, micer Tomás Doria, máximo prestamista del Barrio de Génova, con 4.000 mrs. ${ }^{15}$

Por el número de prestamistas, el peso relativo de la ciudad fue del 41,2\%, mientras que el de la tierra alcanzó el 58,8 \%. Según las nóminas conservadas, en aquella participaron en el reparto 837 vecinos y moradores ${ }^{16}$, a los que hay que añadir los $46^{17}$ de las personas especiales. Esto significa que afectó al 21,4 del conjunto de los vecinos empadronados. Si en vez de la cifra global se tiene en cuenta la media ponderada de cada una de las unidades fiscales, esta se sitúa en el $16 \%$, quedando por encima doce de las collaciones y barrios, de un total de 27 , porque del resto no hay padrones. La horquilla en la que se movieron fue entre el $39,8 \%$ de la collación de Sta. Ma Magdalena y el 2,6 de la de S. Andrés del total de los empadronados.

14. Triano Milán 2013. No se conservan las relaciones de todas las collaciones y pueblos.

15. AMS, Sec. 15, PM, 1429-1430, nº 138, f. 136r.

16. En mi trabajo citado la cifra está errada, debido a un baile de números.

17. Se ha descontado uno que era vecino de Alcalá de Guadaíra, que contribuiría con 30.000 mrs., que también se ha restado del montante de las personas especiales y se ha añadido a los datos de la Campiña. 
En el alfoz, los que participaron fueron 1.262, y el promedio sobre el conjunto de los empadronados del 19,6\%, pero con ciertas diferencias entre las cuatro comarcas que lo integraban: desde el 22,1 \% del Aljarafe y Ribera al 16,6 \% de la Campiña; estando las Sierras de Constantina y de Aroche en el 18,7 y 19,9\%, respectivamente ${ }^{18}$. Por localidades, osciló entre el $60,9 \%$ de Pilas y el $4,1 \%$ de Alcalá del Río.

Estos porcentajes se invierten si lo que se tiene en cuenta es el volumen de lo prestado por cada una de las partes, pues mientras a la ciudad se le asignaron 1.202.900 mrs. (59,6 \%), incluyendo las personas especiales; a la tierra le correspondieron $813.770 \mathrm{mrs}$. $(40,4 \%)$.

En el reparto de dicha cantidad por las collaciones y barrios, la imagen refleja ciertos rasgos de la configuración socioeconómica de Sevilla. Por encima de la media ponderada (29.543 mrs.) solo hay once collaciones y barrios. Entre ellas se encuentran algunas de las más pobladas y situadas en el área de centralidad, como el Salvador, S. Isidoro y los barrios de Castellanos, Francos y de la Mar; pero también collaciones más pequeñas, aunque con presencia de individualidades (S. Nicolás) o de sectores con cierto potencial económico, muy relacionado con la población conversa (Sta. Cruz), o con el río (S. Vicente, Sta. $\mathrm{M}^{\mathrm{a}}$ Magdalena, en este último caso, además de tener muchos vecinos) ${ }^{19}$.

Por lo que respecta al alfoz, la comarca que más aportaba era la Campiña con el 38,4 \%, seguida del Aljarafe y la Ribera, con $30 \%$ y a distancia las Sierras de Constantina, con 17,1 y de Aroche con $14,5 \%$. Si de las comarcas se pasa a las localidades, se confirman rasgos ya conocidos. Con solo once por encima de la media ponderada (20.625 mrs.), entre ellas están todos los grandes pueblos de la Campiña (Alcalá de Guadaíra, Lebrija y Utrera) y los más importantes de las restantes comarcas, destacando por su número los de las Sierras (Alanís, Constantina, Cazalla de la Sierra o Fregenal) y con Sanlúcar la Mayor, por el Aljarafe.

Si de las unidades fiscales pasamos a los individuos, el promedio de lo prestado por los vecinos de Sevilla estuvo en 1.362,3 mrs., mientras que el de los de la tierra quedó en 621,5 mrs., con grandes diferencias entre comarcas, sobre todo porque la Campiña con una media de 1.137 mrs., duplicó a las dos que le seguían, el Aljarafe y Ribera y la Sierra de Constantina, con 559,2 y 567,1 mrs., respectivamente, mientras que la Sierra de Aroche quedó a gran distancia con 335,6 mrs. ${ }^{20}$

Entre esas individualidades, aparte de algunas de las personas especiales, los mayores prestamistas en la ciudad fueron sendos vecinos de S. Nicolás y del Ba-

18. Apéndices 3 y 4. No hay padrones fiscales de estos años, por lo que he recurrido a los que se conservan que van desde 1426 a 1438. Aparte de esto, en la ciudad hay cinco collaciones o barrios y en el alfoz nueve pueblos que no han conservado ninguno de estos años. Por todo ello, estos cálculos hay que entenderlos como una aproximación.

19. Hay que recordar que en estas cifras no están contempladas las cantidades de las personas especiales, porque, salvo excepciones, no se conoce donde residían.

20. En la ciudad, sin contar las personas especiales el promedio queda en 1.129,5 mrs. A modo de referencia, el precio medio de la fanega de trigo en la década de 1420 fue de 27,69 mrs., según la documentación recogida en la serie de Papeles del Mayordomazgo de esos años, por lo que dichas cantidades eran el equivalente a unas 50 fanegas para la ciudad y 22 para los del alfoz. 
rrio de Francos (un trapero), con 15.000 mrs. cada uno, y otros dos de S. Pedro y S. Martín, con 10.000 mrs., respectivamente, que, como he apuntado antes, podrían haber formado parte del grupo de personas especiales. Pero se trata de casos excepcionales, la mayoría se situó entre los 2.000 y los 5.000 mrs. En el extremo opuesto de la escala, la cantidad mínima generalizada fue de $200 \mathrm{mrs}$., aunque en la collación de Santa María Magdalena aparece alguno con 100 mrs. En el alfoz, los máximos prestamistas fueron algunos de los que ya figuraban en la relación de las doblas: el jurado de Alcalá de Guadaíra, con 30.000 mrs., incluido en la nómina de las personas especiales, y dos vecinos de Utrera, el escribano Diego Martínez, con 25.000 mrs., y Juan Alonso de Montesdoca, con 20.000². El resto se encuentra a gran distancia, pues el que le sigue en orden descendente es un vecino de Alcalá de Guadaíra, con 9.000. Por lo demás, en los pueblos la diversidad en las cantidades es considerablemente superior a la de la ciudad, pues va desde la aportación mínima de 150 a los 7.500 mrs. Los escribanos públicos fueron los mayores prestamistas en las villas de Utrera, Sanlúcar la Mayor, Aznalcázar, El Pedroso, Alanís, Cumbres Mayores, Cala y Aracena; en otras se encuentran entre los mayores.

Una vez más, los afectados no estuvieron de acuerdo con el reparto por considerar excesiva la carga, y el concejo procedió a una revisión a la baja de las cantidades asignadas a cada unidad fiscal. De dicha revisión resultó una merma de aproximadamente el $25 \%$ de los dos millones, según se puede observar en el siguiente cuadro resumen que figura en la documentación ${ }^{22}$.

\begin{tabular}{|l|c|}
\hline Monta el $1^{\circ}$ repartimiento de la ciudad & $945.400,0$ \\
\hline Monta el prestido por las 2 ${ }^{\circ}$ copias & $595.300,0$ \\
\hline "Fallesce" & $350.100,0$ \\
\hline & \\
\hline Monta el prestido de la tierra & $783.770,0$ \\
\hline Monta el prestido por el 2" repartimiento & $620.347,5$ \\
\hline "Fallesce" & $163.422,5$ \\
\hline & \\
\hline "Fallesce" la ciudad & $350.100,0$ \\
\hline "Fallescen" las comarcas & $163.422,5$ \\
\hline "Fallesce" las perdonas especiales & $23.000,0$ \\
\hline Puede haber de enmiendas & $25.000,0$ \\
\hline TOTAL "FALLESCE" & $561.522,5$ \\
\hline
\end{tabular}

La rebaja afectó más a la ciudad con el $37 \%$, mientras que para el alfoz quedó en el $21 \%$. Es significativo que de los 39 pueblos que figuran en los cargos, 22

21. AMS, Sec. 15, PM, 1429-1430, f. 119.

22. AMS, Sec. 15, PM, 1429-1430, no 318 , f. 22 v 
mantuvieran las cantidades inicialmente asignadas, más otras dos con apenas reducción, mientras que en la ciudad solo fueron cinco, más otras dos que apenas la experimentaron. Pero las diferencias entre las doce del alfoz fueron importantes pues las rebajas oscilaron entre el $76 \%$ (Pilas) y el 4,9\% (Sanlúcar la Mayor). De nuevo, entre ellas figuran la mayor parte de las que se encontraban por encima de la media por volumen de lo prestado. A parte de esto, en el alfoz se dieron los dos únicos casos en que en vez de reducir sus cantidades, lo que hicieron fue incrementarlas: El Pedroso pasó de 3.250 a 9.750 mrs. y La Puebla del Infante, de 2.025 a 5.025 mrs., ambas villas en la Sierra de Constantina. En la ciudad las diferencias entre collaciones y barrios fueron prácticamente idénticas a las del alfoz. Sin embargo, aquí las reducciones más importantes, por encima del $50 \%$, afectaron a collaciones de tipo medio, algunas periféricas y con vecindarios con niveles económicos bajos, mientras que entre el 33 y el $50 \%$ se encuentran las de mayor nivel socioeconómico ${ }^{23}$.

Pero lo extraño de esta operación es que dicha reducción no fue compensada para poder llegar a los dos millones de maravedís. En los nuevos cargos hechos a los recaudadores solo figuran las cantidades resultantes de dicha rebaja:

\begin{tabular}{|l|l|r|r|r|}
\hline \multicolumn{1}{|c|}{ Recaudadores } & \multicolumn{1}{c|}{ Distrito } & \multicolumn{1}{c|}{$1^{\circ}$ cargo } & \multicolumn{1}{c|}{$2^{\circ}$ cargo } & quitado \\
\hline Manuel González de Ocaña & Ciudad & 202.300 & 94.050 & 108.250 \\
\hline Juan Rodríguez de Illescas & Ciudad & 298.400 & 196.400 & 102.000 \\
\hline Juan González de Sevilla & Ciudad & 142.900 & 105.000 & 37.900 \\
\hline Pedro González de Córdoba & Ciudad & 301.800 & 199.850 & 102.350 \\
\hline Ruy Sánchez, jurado & S. Aroche & 113.775 & 100.725 & 13.045 \\
\hline especiales & & 287.500 & 254.500 & 23.000 \\
\hline Fernán Martínez de Sevilla & Aljarafe Ribera & 235.420 & 205.842 & 29.576 \\
\hline Alfonso Fernández de Escobar & S. Constantina & 134.400 & 122.030 & 12.370 \\
\hline Andrés Rodríguez & Campiña & 300.175 & 191.750 & 108.425 \\
\hline TOTAL & & 2.016 .670 & 1.503 .147 & 536.916 \\
\hline
\end{tabular}

La reducción de las cantidades se acompañó con el incremento del número de vecinos a los que demandar el préstamo. Por todo ello, ahora, las cantidades mínimas fueron $50 \mathrm{mrs}$. en la ciudad, con una única excepción de $30 \mathrm{mrs}$. para un vecino del Barrio Nuevo, mientras que en el alfoz los hubo que participaron con solo $5 \mathrm{mrs}$. La rebaja también afectó a las cifras máximas, aunque se mantuvo alguna $^{24}$. Todo lo cual supuso incrementar el colectivo obligado a contribuir en el empréstito, que en la ciudad alcanzó a 1.912 vecinos $^{25}$, más las 46 personas

23. AMS, Sec. 15, PM, 1429-1430, n 318, f. 21r-22v.

24. Apéndice 4. Los dos vecinos de Utrera a los que en el primer cargo se le asignaron 25.000 y 20.000 mrs., respectivamente; en el segundo se les rebajaron a 12.000 y 8.000 (AMS, Sec. 15, PM, $1429-1430, \mathrm{n}^{\circ} 138$, f. $\left.279 \mathrm{v}\right)$.

25. Apéndice 3. 
especiales, mientras que en la tierra, a falta de los datos de nueve localidades, fueron 2.899 .

En cuanto al peso que hubiera soportado cada una de las partes, a la ciudad se le repartieron 859.600 mrs., por 650.353 al alfoz, en ambos casos incluyendo lo correspondiente a las personas especiales. Lo que significa que, con las salvedades ya señaladas por la falta de algunas nóminas, el promedio en la ciudad habría sido de 439 mrs. $^{26}$, por 224,3 mrs. para los del alfoz. Desglosado por comarcas, los que más hubieran contribuido habrían sido, de nuevo, los de la Campiña con 509,7 mrs., seguidos de los del Aljarafe y la Ribera, con 204,6, los de la Sierra de Aroche, con 159,6 y los de la de Constantina, con 147,5 mrs.

De todas formas, estas cifras tuvieron que ser rápidamente rectificadas. No he encontrado información sobre cómo se resolvió, salvo que la revisión estaba planteada a mediados de diciembre; que posiblemente fuese realizada por Nuño López de Saldaña ${ }^{27}$; y que afectó tanto a la ciudad como al alfoz. Lo que sí pone de manifiesto el cuadro adjunto, que refleja los importes de los dos cargos ya conocidos y del cargo final de cada recaudador una vez incorporado el reparto de las cantidades "fallecidas", es que no fue igual para todas las collaciones y pueblos $^{28}$ :

\begin{tabular}{|c|c|c|c|c|}
\hline Recaudadores & Distrito & $1^{\text {er }}$ cargo & $2^{\circ}$ cargo & Cargo final \\
\hline Juan González de Sevilla & Ciudad & 142.900 & 105.000 & 105.000 \\
\hline Pedro González de Córdoba & Ciudad & 301.800 & 199.850 & 199.850 \\
\hline Manuel González de Ocaña & Ciudad & 202.300 & 94.050 & 95.550 \\
\hline Juan Rodríguez de Illescas & Ciudad & 298.400 & 196.400 & 619.110 \\
\hline Ruy Sánchez & $\begin{array}{l}\text { Sierra Aroche } \\
\text { Campiña } \\
\text { Personas especiales }\end{array}$ & $\begin{array}{l}113.775 \\
287.500\end{array}$ & $\begin{array}{l}100.725 \\
264.500\end{array}$ & $\begin{array}{l}100.725 \\
133.225 \\
264.500\end{array}$ \\
\hline Fernán Martínez de Sevilla & Aljarafe y Ribera & 235.420 & 205.842 & 325.757 \\
\hline Alfonso Fernández Escobar & Sierra Constantina & 134.122 & 122.030 & 122.030 \\
\hline Andrés Rodríguez & Campiña & 300.175 & 191.750 & 191.750 \\
\hline Alfonso López & Sierra Constantina & & & 57.920 \\
\hline Juan García de Sevilla & Sierra Aroche & & & 58.160 \\
\hline TOTAL & & 2.016 .670 & 1.480 .147 & 2.273 .852 \\
\hline
\end{tabular}

26. Sin las personas especiales quedó en 311,2 mrs.

27. La fecha se deduce de la mención a dicho reparto en un mandamiento a los contadores de Sevilla fechado el 14 de diciembre (AMS, Sec. 15, PM, 1429-1430, n 138, f. 297r). En cuanto a que el autor fuese el tesorero es lo que manifiestan diversos vecinos que se consideraban agraviados (AMS, Sec. 15, PM, 1429-1430, no 138, ff. 286r-287r).

28. AMS, Sec. 15, PM, 1429-1430, no 138, ff. 292r-293r. Desconozco el criterio seguido para el reparto de la ciudad entre los cuatro recaudadores, pues no fue por áreas geográficas, y las collaciones y barrios eran de distintos niveles poblacionales y socioeconómicos. 
Como se puede ver, el incrementó afectó a todas las comarcas de la tierra de Sevilla en distinta proporción:

Campiña. ..........

$69.5 \%$

Aljarafe y Riera. ..... $\quad 58.3 \%$

Sierra de Aroche .... $57.7 \%$

Sierra de Constantina. . $\quad 47.5 \%$

En el caso de la ciudad, el incremento figura solo en el cargo de Juan Rodríguez de Illescas, nada menos que el 215,2 \%. Dado que en el grupo de collaciones y barrios de los que era recaudador (barrios de Castellanos, de la Mar, Omnium Sanctorum, S. Andrés, S. Gil, S. Martín, S. Miguel, S. Nicolás, Santa Cruz y Triana) los hay de muy diferentes niveles socioeconómicos y poblacionales, cabe la posibilidad de que se le cargasen incrementos de otros barrios y collaciones, como había ocurrido en el alfoz con Ruy Sánchez. Ante la duda, solo cabe tomar la ciudad en su conjunto; en este caso, el incremento habría sido del 58,4\%, que está en la media de las comarcas del alfoz.

Este reajuste trajo consigo el de los recaudadores y de los cargos, pues, como se ha podido comprobar en el cuadro, ahora aparecen dos nuevos, el escribano del concejo Alfonso López y Juan García de Sevilla, como recaudadores de los incrementos de las Sierras de Constantina y Aroche, respectivamente; además a Ruy Sánchez, que ya lo era de las personas especiales y de la Sierra de Aroche se le suma ahora el incremento de la Campiña.

Al no conservarse las nóminas de las unidades fiscales no es posible comparar este reparto definitivo con el inicial, y hay que limitarse a los datos globales. En cuanto a la participación de la ciudad y de su alfoz, las nuevas y definitivas cifras fueron 1.254 .010 y $1.019 .842 \mathrm{mrs}$., respectivamente, lo que supone unos porcentajes de 55,2 y $44,8 \%$, por lo que la ciudad rebajaba en cuatro puntos su participación. En cuanto al alfoz, aunque se mantuvieron las diferencias entre las cuatro comarcas, tuvo lugar un reajuste, pues la Campiña, con el 34,8 \%, rebajaba seis puntos, mientras que el Aljarafe y la Ribera, con el $32 \%$, incrementaba cuatro puntos, y las dos Sierras, a gran distancia de las precedentes, incrementaban un punto cada una, la de Constantina con el 17,6 \% y la de Aroche con el 15,6 \%.

Como era normal en este tipo de procedimientos para responder a las exigencias de los monarcas, sus premuras chocaron con la lentitud de la respuesta. En esta ocasión, sobre todo los cambios de criterio y las sucesivas quejas, hicieron que se tardase meses en recaudar la cantidad solicitada ${ }^{29}$.

29. Una parte importante de la documentación conservada no está fechada, lo que dificulta seguir la secuencia temporal. En relación con otras ciudades y a tenor de lo publicado, observo notables desfases temporales. En el caso de Murcia, la carta de poder está fechada el 22-XI-1429, y en Burgos se estaba negociando en el mismo mes, aunque los acuerdos con particulares pudieran ser anteriores y, en ambos casos, entrado el año siguiente todavía se estaba negociando o exigiendo su recaudación 
Los primeros documentos conocidos son las cartas de poder a favor de Nuño López de Saldaña, recaudador del préstamo por el rey, fechadas el 20 de junio ${ }^{30}$, pero hasta el mes de agosto no hay datos sobre el proceso. En ese momento estaba constituida la comisión creada por el cabildo para gestionar la recaudación ${ }^{31}$, y ya se había tomado la decisión de implicar a los vecinos y moradores, pues se escribió a las villas del alfoz para que enviasen sus representantes a Sevilla, con el fin de tratar del reparto ${ }^{32}$. Este se fue configurando a lo largo de septiembre, y estaba cerrado el 24 de dicho mes.

En el cabildo del citado día se debatió sobre el procedimiento para recaudarlo y la conveniencia de contar con recaudadores. Se acordó que fuesen tres para la ciudad y cuatro para las comarcas del alfoz, delegando en la comisión su nombramiento, así como la expedición de los correspondientes recudimientos ${ }^{33}$. Además, se pasó toda esta información a los contadores de concejo, que fueron los responsables institucionales del control de legalidad de todo el proceso.

En los primeros días de octubre estaban nombrados los recaudadores, que ya era cuatro en la ciudad ${ }^{34}$, y asignados los cargos, pues se firmaron los recudimientos y cartas de obligación los días siete y ocho de octubre ${ }^{35}$, comenzando de inmediato la recaudación ${ }^{36}$. Sin embargo, a pesar de las exigencias del monarca,

(Abellán Pérez 1984, pp. 397-398. Archivo Municipal de Murcia, Actas Capitulares, libro 52, ff. 53v-54r. Guerrero Navarrete 1999, p. 100).

30. AMS, Sec. 15, PM, 1429-1430, nº 138, ff. 3r-4v.

31. La componían un alcalde mayor, Juan Fernández de Mendoza, y tres veinticuatros, el propio Nuño López de Saldaña, Diego López de Estúñiga y Diego Ortiz (AMS, Sec. 15, PM, 1429-1430, nº 138, f. 5).

32. Collantes de Terán Delorme 1980, p. 244.

33. AMS, Sec. 15, PM, 1929-1930, nº 138, f. 5v. Tenían la doble misión de recaudar el dinero de las personas obligadas a participar en el préstamo y luego reintegrarles las cantidades prestadas, cuando por la administración real se devolviesen los dos millones de maravedís (ff. 8r-9v). La remuneración que figura en estos documentos fue de $25 \mathrm{mrs}$. al millar, si bien parece que posteriormente se rebajó a 15 (f. 295r). A los recaudadores que les correspondió contribuir en el préstamo, se les eximió de dicha participación (f. 297r).

34. AMS, Sec. 15, PM, 1929-1930, nº 138, f. 5v. Los de la ciudad fueron Manuel González de Ocaña, v $v^{\circ}$ Salvador, que sería mayordomo del concejo en 1437-1438 y 1441-1442; Juan Rodríguez de Illescas, v $v^{\circ}$ Sta. Cruz; Juan González de Sevilla, vo Salvador; y Pedro González de Córdoba, vo S. Martín. Los del alfoz fueron Fernán Martínez de Sevilla, $v^{\circ} \mathrm{S}$. Miguel y criado de Álvaro de Luna, para el Aljarafe y la Ribera; el jurado Ruy Sánchez, v S. Román, para la Sierra de Aroche y para las personas especiales; el jurado Alfonso Fernández de Escobar, v $v^{\circ}$ S. Julián, para la Sierra de Constantina; y el cirujano del concejo Andrés Rodríguez, vo Salvador y mayordomo en 1422-1423, para la Campiña. Como se ha visto, en la fase final aparecen otros dos, Juan García de Sevilla y el jurado y lugarteniente del escribano mayor del cabildo Alfonso López, $v^{\circ} \mathrm{S}$. Julián, que actuaron respectivamente, en las Sierra de Aroche y de Constantina. Varios de ellos figuran en las relaciones de las collaciones, por lo que es posible conocer la cantidad con la que habrían contribuido si no hubiesen quedados exentos. Manuel González Ocaña debía haber prestado 1.500 mrs. en el primer cargo, y se le rebajó a 1.200 mrs. en el segundo (AMS, Sec. 15, PM, 1929-1930, nº 138, ff. 48r, 164r); el cirujano Andrés Rodríguez, 1.500 y 1.300, respectivamente (ff. 48r, 164r); Juan Rodríguez de Illescas, 3.000 en los dos cargos (ff. 57r, 182r); y Pedro González de Córdoba, 2.000 y 1.000, respectivamente (ff. 61r, 192r).

35. AMS, Sec. 15, PM, 1429-1430, $\mathrm{n}^{\circ} 138$, ff. 6r-9v.

36. Aparte de que al recaudador Manuel González de Ocaña se le cargaron 1.500 mrs. que había cobrado de varias personas correspondientes al primer cargo (AMS, Sec. 15, PM, 1429-1430, $\mathrm{n}^{\circ}$ 138, 
que urgía la entrega del préstamo ${ }^{37}$, aquella se vería frenada debido a la protesta generalizada, que obligó a efectuar el nuevo reparto y a la redacción de los consiguientes nuevos cargos a los recaudadores, al tiempo que se revisaban las cantidades asignadas a vecinos concretos que se sentían agraviados ${ }^{38}$. Los recudimientos están fechados el dos de diciembre ${ }^{39}$. Según esto, sería en el citado mes cuando se estaría efectuando el grueso de la recaudación, porque el 14, ya se debía estar haciendo el reajuste con las cantidades "fallecidas"

Estas últimas actuaciones coincidieron con un cambio en el recaudador designado por Juan II. Ante las misiones que había encomendado a Nuño López de Saldaña, le sustituyó por el jurado sevillano Bartolomé Fernández de Villalán ${ }^{41}$. Dicho nombramiento fue cuestionado por una parte de los capitulares con el argumento de que se había dado el cargo de los dos millones al tesorero y se había ordenado a los recaudadores que acudiesen a él con las cantidades que fuesen percibiendo, por lo que el nuevo nombramiento obligaba a anular el citado cargo cuando ya se estaban cobrando cantidades, y a rehacer las cartas de recudimiento. Por su parte, el jurado se quejó de que el cabildo le hubiese cargado los dos millones, a pesar de que los recaudadores habían ido efectuando entregas al tesorero y las había invertido por orden regia. Ante lo cual, solicitó que pudiese tomar cuenta de lo que ya se había pagado, incluido al propio tesorero, y que solo se le cargase lo que restaba. La respuesta del monarca a estas quejas no llegaría hasta mediados de enero. En ella ordenó al concejo que permitiese a Bartolomé Fernández de Villalán ajustar con los recaudadores y el tesorero las cantidades entregadas y los gastos efectuados, y que solo se le cargase lo que restase por entregar del total del préstamo ${ }^{42}$.

Para complicar algo más la gestión, el mismo día en el que el cabildo ordenó a los recaudadores acudir al jurado con lo que recaudasen -27 de enero de 1430-, Juan II volvió a nombrar a Nuño López de Saldaña, debido a la mala gestión de aquel, porque

f. 141r), según una carta del 18 de noviembre ya se habían efectuado importantes gastos en la compra de trigo y harina y en transportes a Guadalajara y otros lugares (AMS, Sec. 15, PM, 1429-1430, $\mathrm{n}^{\circ}$ 138, f. 16r-v).

37. Santa María de Fresdelval, 14-10-1429 (AMS, Sec. 15, PM, 1429-1430, nº 138, ff. 15v-16r).

38. AMS, Sec. 15, PM, 1429-1430, nº 138, ff. 129r, 141r, 159r, 178r.

39. AMS, Sec. 15, PM, 1429-1430, nº 138, ff. 122r-v, 158r-v, 177r-v, 204r-v, 234r-v, 253r-v, $243 r-274 r$.

40. Estas fechas se compaginan mal con la afirmación que hace Juan II en carta dirigida al concejo de Burgos el 30 de noviembre, en la cual afirma que Sevilla le ha enviado los dos millones de maravedís (Archivo Municipal de Burgos, Acta Capitular de 6-12-1429, LA6, fol. 90v, cit. MacKay 2006, p. 130).

41. La carta de nombramiento está fechada el 18 de noviembre de 1429 , pero no se vio en el cabildo hasta un mes después, el 19 de diciembre (AMS, Sec. 15, PM, 1429-1430, nº 138, fol. 16). Bartolomé Fernández de Villalán también fue el recaudador de los pedidos y monedas de esta año y posteriores (Collantes de Terán Delorme 1980, pp. 245, 277. Kirschberg Schenck 2011, n 224, 363, $571,598-600)$.

42. 14-1-1430 (AMS, Sec. 15, PM, 1429-1430, n 138, ff. 10r-v, 17r-v). 
non ha puesto la diligençia que complía en cobrar de vosotros los dichos dos cuentos de maravedis, por cabsa de lo qual, dis que se ha enbargado lo que yo así encomendé e mande al dicho mi tesorero que fesiese, de que a mi se ha seguido e sigue deseruiçio ${ }^{43}$.

El mandamiento subsiguiente a los recaudadores para que acudiesen con el dinero a Nuño López de Saldaña está fechado el ocho de febrero ${ }^{44}$.

En los meses posteriores los recaudadores fueron entregando remesas al tesorero, pero las quejas y los problemas llevaron al cabildo a designar a los veinticuatros Fernán Ruiz Cabeza de Vaca y Alfonso Fernández de Santillán, para que, junto con los contadores, se encargasen de gestionar el proceso final de la recaudación. Su cometido fue resolver las quejas de los agraviados, fiscalizar las cuentas de los recaudadores y de Fernández de Villalán y exigirles el cumplimiento de sus obligaciones. Además, se les encomendó controlar la posterior devolución de las cantidades prestadas ${ }^{45}$. Es en estos documentos de rendiciones de cuentas donde aparecen los dos nuevos recaudadores, aunque con cargos mucho más modestos como se ha visto. El resumen final de las distintas fases se sintetiza en el siguiente cuadro ${ }^{46}$.

\begin{tabular}{|l|r|r|r|r|r|c|}
\hline \multicolumn{1}{|c|}{ Recaudadores } & \multicolumn{1}{c|}{ Cargos } & Quiebras & \multicolumn{1}{c|}{ A pagar } & \multicolumn{1}{c|}{ Pagado } & Alcances & Total \\
\hline $\begin{array}{l}\text { Juan González } \\
\text { de Sevilla }\end{array}$ & 105.000 & 15.295 & 89.705 & 86.555 & 3.150 & 89.705 \\
\hline $\begin{array}{l}\text { Pedro González } \\
\text { de Córdoba }\end{array}$ & 199.850 & & 150.506 & 150.507 & 9.450 & 159.957 \\
\hline $\begin{array}{l}\text { Manuel González } \\
\text { de Ocaña }\end{array}$ & 95.550 & 12.300 & 83.250 & 81.213 & 2.037 & 83.250 \\
\hline $\begin{array}{l}\text { Juan Rodríguez } \\
\text { de Illescas }\end{array}$ & 619.110 & 70.031 & 549.078 & 530.050 & 19.028 & 549.078 \\
\hline Ruy Sánchez & 498.725 & 66.000 & 432.725 & 416.960 & 15.765 & 432.725 \\
\hline $\begin{array}{l}\text { Fernán Martínez } \\
\text { de Sevilla }\end{array}$ & 325.757 & 27.900 & 297.857 & 284.440 & 13.417 & 297.857 \\
\hline $\begin{array}{l}\text { Alfonso Fernández } \\
\text { Escobar }\end{array}$ & 122.030 & 16.865 & 105.165 & 80.400 & 24.765 & 105.165 \\
\hline Andrés Rodríguez & 191.750 & 13.003 & 178.747 & 173.554 & 9.286 & 182.840 \\
\hline
\end{tabular}

43. AMS, Sec. 15, PM, 1429-1430, $\mathrm{n}^{\circ} 138$, ff. 16v-19v.

44. AMS, Sec. 15, PM, 1429-1430, no 138, ff. 15r-19v.

45. 12-4-1430 (AMS, Sec. 15, PM, 1429-1430, n 138, ff. 286r-287r).

46. AMS, Sec. 15, PM, 1429-1430, nº 138, ff. 289r-291v, 292r-294v.

47. Sobre su cuenta y posible quiebra figura la siguiente nota: "E por quanto non ha fenesçido su cuenta, cobrad dél, con los mrs. que por recabdos çiertos vos mostrare que vos ha pagado, clUdvj mrs. j c ${ }^{o}$ para complimiento de los dichos dos cuentos de mrs. E lo quel dicho Pero Gonzales demás deue de los dichos mrs. del dicho su cargo a los de pagar a Seuilla".

48. Dio en quiebra 8.910 mrs., más 4.093 mrs. que debía retener para entregar al concejo. En 1445, aun le reclamaba Sevilla esta cantidad (AMS, Sec. 15, PM, 1444-1445, n 1.261, f. 51v). 


\begin{tabular}{|l|r|r|r|r|r|c|}
\hline \multicolumn{1}{|c|}{ Recaudadores } & \multicolumn{1}{c|}{ Cargos } & Quiebras & \multicolumn{1}{c|}{ A pagar } & Pagado & Alcances & Total \\
\hline Alfonso López & 57.920 & & 57.920 & & 57.920 & 57.920 \\
\hline $\begin{array}{l}\text { Juan García } \\
\text { de Sevilla }\end{array}$ & 58.160 & 3.114 & 55.046 & 53.166 & 1.880 & 55.046 \\
\hline TOTAL & 2.273 .852 & 224.508 & 1.999 .999 & 1.856 .845 & 156.698 & 2.013 .543 \\
\hline
\end{tabular}

Aunque en el margen de la copia en la que los diputados hacían el balance hay una anotación en la que se aclara que no la habían firmado los contadores y, en consecuencia, estaba pendiente de que ellos revisasen las cuentas y exigiesen a los recaudadores la documentación necesaria para recibirles las quiebras, en la relación final -sin fecha-, en la que se registra lo pagado y los alcances que se les reconocían, no se observa cambio alguno en las cantidades. Es más, en una de las copias hay otra anotación marginal en la cual se dice que por ella se recibió el tesorero de los dos millones de maravedís y firmó la carta de pago, fechada en Sevilla el dos de mayo de $1431^{49}$. Sin embargo, el documento en el que los diputados le remitieron la liquidación es del 17 del citado mes, y todavía el 4 de junio el concejo ordenaba al alguacil mayor que actuase contra los recaudadores que no cumpliesen con las cantidades que les habían sido cargadas ${ }^{50}$.

Por lo que se refiere al superávit de $13.543 \mathrm{mrs} .5 \mathrm{~d}$., un documento sin fecha ni firma, pero que posiblemente redactara alguno de los escribanos que intervinieron en esta fase final, indica que la citada cantidad correspondía a los $4.093 \mathrm{mrs}$. que los capitulares ordenaron al recaudador Andrés Rodríguez que los retuviese para Sevilla, y los restantes 9.450 eran los que pretendía quedarse el recaudador Pedro González de Córdoba en concepto de costas y salario. Dicha pretensión le había sido denegada, porque el importe de los salarios se cargaba sobre las cantidades entregadas al tesorero y no lo pagaba la ciudad. Además, el redactor manifestaba que los diputados dieron por buenas las quiebras presentadas por los recaudadores, a la espera de que los contadores las aprobasen, para así rematar la cuenta con Nuño López de Saldaña, al que solo reconocieron los 2.000.000 mrs. del préstamo, y terminaba con la siguiente consideración:

E los jx $U \operatorname{cccc}^{\circ} l$ maravedís que Pedro Gonzáles de Córdoua da en su breue relación de cuenta que dio, dise e pone que los ha de aver de salario e costas. Estos ha de pagar él de razón a Seuilla, quando bien librase, por quanto Seuilla non ha de pagar maravedis algunos de salario a él nin a los otros recaudadores, que asý fue la conpusiçión, saluo el dicho tesorero los ha de contentar de salario. Nin menos, resçebir maravedis de costas, por quanto el recaudador que lieua salario non ha de poner en cuenta costas nin burlas. E asý están los dichos xiij $U d x$ x iij mrs. $v$ d. de alcance que se deuen a Seuilla, para quando ella los mandare pagar con los otros maravedis que se sacarán, si Dios quiere, de las quiebras de burla que los

49. AMS, Sec. 15, PM, 1432-1433, nº 209.

50. AMS, Sec. 15, PM, 1429-1430, no 138, ff. 289r-291v, 292r-293v. 
recabdadores dieron. E yo juro en mi conçiençia que avnque alguna cosa dello sea verdad, que lo más es todo burla de tantas quiebras ${ }^{51}$.

Una vez cerrado el proceso de recaudación, debía abrirse el de devolución de las cantidades prestadas. Sin embargo, se fueron produciendo sucesivos aplazamientos. Cuando Juan II solicitó el préstamo se comprometió a devolverlo sobre ciertas rentas de Sevilla y su tierra por los tercios del año $1430^{52}$; pero ya el 10 de noviembre de 1429 comunicó al concejo que se haría en 1431, y se cargaría sobre las alcabalas de las mercaderías, de la Alhóndiga, carnicerías, vino, pescado fresco y salado, aceite, varas, fruta verde y seca ${ }^{53}$. Llegado dicho año, en el mes de enero, el rey ordenó a los diputados designados por el concejo para controlar el proceso, que entregasen al doctor Pedro González Hontiveros los repartimientos y las copias de las cantidades pagadas por cada vecino, con el fin de que los estudiase e informase ${ }^{54}$ y a principios de marzo, el día siete, firmó un nuevo aplazamiento hasta el año siguiente, debido a los gastos generados por la campaña contra el Reino de Granada. En el margen de la copia de esta carta hay la siguiente anotación: "Veno el anno de xxxij, pero nunca se pagaron los dichos dos cuentos fasta que Dios quiera e el rey nuestro sennor".

A mediados de 1433 el concejo recibió un escrito para que enviase una serie de documentos porque el monarca estaba dispuesto a abonar la deuda ${ }^{55}$. A comienzos de julio fue enviado el procurador mayor y veinticuatro Luis Fernández del Marmolejo, que se unió en la corte al tesorero y veinticuatro Nicolás Martínez de Medina/Sevilla y al jurado García Sánchez de Sevilla, quienes negociaban otros temas relacionados con la hacienda regia. Se entrevistaron con el tesorero Fernán López de Valladolid, a quien fueron remitidos por el monarca, y este les hizo saber que el rey no tenía dinero para pagar ${ }^{56}$.

Entonces se propuso algo que por la forma en que se expone debía de haber sido planteado con anterioridad: la recuperación por parte del concejo sevillano de la villa de Arcos a cuenta de los dos millones. Se comunicó la propuesta al monarca, y este aceptó con la condición de que, además de la citada cantidad de la deuda, la ciudad abonase 20.000 florines (1.070.000 mrs.). Nicolás Martínez de Medina/ Sevilla le respondió al relator que no lo aceptaba por considerarla desproporcionada. Sin embargo, cuando este se marchó y el tesorero quedó a solas con el jurado, le comentó que su impresión era que nunca se devolvería el préstamo, y puesto que ya estaban entregados los dos millones, no era tan desorbitada la petición de los 20.000 florines, dado que se obtendrían de una imposición sobre la carne ${ }^{57}$.

51. AMS, Sec. 15, PM, 1429-1430, $\mathrm{n}^{\circ}$ 138, f. 295r. En una nota marginal, se indica que el salario era de 15 mrs. al millar.

52. AMS, Sec. 15, PM, 1429-1430, nº 138, f. 4 v.

53. AMS, Sec. 15, PM, 1429-1430, n ${ }^{\circ} 138$, f. $11 \mathrm{r}-\mathrm{v}$.

54. AMS, Sec. 15, PM, 1429-1430, nº 138, f. 296.

55. AMS, Sec. 15, PM, 1432-1433, nº 186; 1433-1434, nº 209.

56. AMS, Sec. 15, PM, 1432-1433, nº 26, 27. Ambos documentos carecen de año. En el Catálogo figura suplido el año 1432; sin embargo, por otros documentos, corresponde a 1433.

57. El valor del florín en MacKay 2006, p. 183. AMS, Sec. 15, PM, 1432-1433, nº 203. 
Esta negociación no siguió adelante, pues al año siguiente los capitulares enviaron al veinticuatro Gonzalo de Cuadro con un escrito en el que después de invocar los servicios prestados, volvieron a demandar el pago de la deuda, y como alternativa la ya mencionada recuperación de Arcos, aunque tampoco tuvieron éxito $^{58}$. En las Cortes de Valladolid de 1442, los procuradores del reino volverían a solicitar la devolución, y Sevilla sería una de las ciudades implicadas, porque creo que en 1446 todavía se estaba reclamando ${ }^{59}$.

\section{BIBLIOGRAFÍA CITADA}

Abellán Pérez, Juan (1984), Colección de documentos para la historia del Reino de Murcia. Documentos de Juan II, Murcia.

Collantes de Terán Delorme, Francisco (1980), Inventario de los Papeles del Mayordomazgo del siglo XV, t. II, 1417-1431. Sevilla.

Collantes de Terán Sánchez, Antonio (2000), "Moneda y cambios en la Sevilla bajomedieval”, en Antonio M. Bernal, Dinero, moneda y crédito en la Monarquía Hispánica, Madrid, pp. 59-82.

Collantes de Terán Sánchez, Antonio (2009), “El empréstito en la Sevilla bajomedieval", Historia, Instituciones, Documentos, 36, pp. 137-159.

Cortes de los antiguos reinos de León y de Castilla (1866), Madrid.

Gálvez Gambero, Federico y Triano Milán, Juan Manuel (2016/2), "La negociación de lo extraordinario en tiempos de Isabel I (1474-1504)", Tiempos Modernos, 33, pp. 294-325.

Guerrero Navarrete, Yolanda (1999), "El déficit de la Hacienda municipal burgalesa en el siglo XV: hacia una evaluación socio-económica y socio-política", Edad Media. Revista de Historia, 2, pp. 81-112.

Kirschberg Schenck, Deborah (2011), Catálogo de los Papeles del Mayordomazgo del siglo XV. III, 1432-1442, Sevilla.

MacKay, Angus (2006), Moneda, precios y politica en la Castilla del siglo XV, Granada-Sevilla.

Ortego Rico, Pablo (2012), "Las riquezas de la Iglesia al servicio del poder monárquico: los empréstitos eclesiásticos en la Castilla del siglo XV", En la España Medieval, 35, pp. 145-176.

58. AMS, Sec. 15, PM, 1432-1433, n 203. En el caso de que se hubiese devuelto Arcos, hubiera quedado pendiente la recuperación de los dos millones por quienes lo habían prestado, por lo que los representantes de Sevilla acompañaron la solicitud referida a Arcos con la petición al monarca de que les autorizase a echar una imposición sobre las rentas de la ciudad para reunir la citada cantidad.

59. Olivera 1998, p. 225. Cortes 1866, pp. 312, 405. Gálvez Gambero y Triano Milán 2016 , p. 298. Tanto en Burgos como en Murcia está documentado el retraso o incumplimiento de la devolución (Guerrero Navarrete 1999, p. 100. Abellán Pérez 1984, p. 419). Respecto a la reclamación de Sevilla en 1446 (AMS, Sec. 10, 16-IX-1446, fol. 38v), creo que se refiere a la de 1429, pues aunque parece que en el empréstito de 1444 también se repartieron dos millones, todavía no estaba cerrada la cuenta en el citado mes y año, que es cuando se tomó el acuerdo mencionado (Collantes de Terán Sánchez 2009, pp. 139, 151). 
Ortego Rico, Pablo (2015), Poder financiero y gestión tributaria en Castilla: los agentes fiscales en Toledo y su reino (1429-1504), Madrid.

Triano Milán, José Manuel (2013), "El arzobispado de Sevilla y el obispado de Cádiz: imagen de un gran partido fiscal en la recaudación del pedido regio", Historia. Instituciones. Documentos, 40, pp. 361-395).

Triano Milán, José Manuel (2018), La llamada del rey y el auxilio del reino. Del pedido regio a las contribuciones de la Santa Hermandad (1406-1498), Sevilla. 